Philosophie ANTIQUE

\section{Philosophie antique}

Problèmes, Renaissances, Usages

$17 \mid 2017$

Platon et la politique

\title{
Socrate « oikiste » et Apollon exégète
}

\section{Xavier Brouillette}

\section{(2) OpenEdition}

\section{Journals}

Édition électronique

URL : https://journals.openedition.org/philosant/279

DOI : 10.4000/philosant.279

ISSN : 2648-2789

\section{Éditeur}

Éditions Vrin

\section{Édition imprimée}

Date de publication : 1 novembre 2017

Pagination : $31-35$

ISBN : 978-2-7574-1807-9

ISSN : 1634-4561

\section{Référence électronique}

Xavier Brouillette, « Socrate « oikiste » et Apollon exégète », Philosophie antique [En ligne], 17 | 2017, mis en ligne le 01 novembre 2018, consulté le 03 décembre 2022. URL : http://

journals.openedition.org/philosant/279; DOI : https://doi.org/10.4000/philosant.279

\section{(c) (i) $9 \odot$}

Creative Commons - Attribution - Pas d'Utilisation Commerciale - Pas de Modification 4.0 International - CC BY-NC-ND 4.0

https://creativecommons.org/licenses/by-nc-nd/4.0/ 


\section{SOCRATE «OIKISTE » ET APOLLON EXÉGÈTE}

Xavier Brouillette

Collège du Vieux Montréal

RÉsumÉ. En République, II, 369a, Socrate choisit de rechercher la justice dans la polis plutôt que dans l'âme humaine. Pour ce faire, il propose de construire une cité en paroles. Cette construction prendra la forme d'une entreprise de colonisation qui aura son terme au livre IV, $427 \mathrm{c}$. Le présent article s'intéresse à la manière dont Platon s'y est pris pour réaliser cette fondation. L'analyse que nous proposons vise principalement à montrer comment Platon s'inspire du modèle historique de la colonisation pour construire cette cité-modèle. La nomination d'un oikistes, la mise en place de lois fondamentales ainsi que la consultation de l'Apollon delphique assurent à la cité une fondation idéale dans laquelle, parce qu'idéalement fondée, la justice pourra être visible.

Summary. In Republic, II, 369a, Socrates chooses to seek justice in the polis rather than in the human soul. To do this, he proposes to build a city in speech. This construction will consist of a settlement project which will have its term in book IV, 427c. This article focuses on how Plato went about achieving this foundation. The analysis we propose is primarily intended to show how Plato uses the historical pattern of colonization to build his model city. The appointment of an oikistes, the promulgation of fundamental laws and the consultation of the Delphic Apollo, provide the city an ideal foundation in which, because ideally founded, justice will be found. 

L'objectif de la République est de définir la justice et d'observer dans quelle mesure elle est souhaitable ${ }^{1}$. Socrate et ses interlocuteurs portent d'abord leur attention sur l'âme, où la structure des vertus est dans un premier temps traquée, mais en vain. Ils conviennent alors de rechercher la justice dans l'ensemble plus vaste qu'est la cité, espérant qu'elle y soit davantage visible (II, 368c-369a). Cette méthode a retenu l'attention des chercheurs, principalement au sujet des rapports entre la structure de l'âme et la structure de la cité2. Surtout, par cette stratégie, Platon met au cœur de la République une réflexion sur la cité et son fondement en proposant de construire « en paroles » une cité (II, 369c).

Le choix de déplacer la réflexion vers la polis est loin d'être anodin, car l'idée de créer une cité n'avait rien de radicalement nouveau, ni de particulièrement étrange à l'époque de Platon. Créer des institutions politiques était une pratique courante, ritualisée, documentée et largement diffusée ${ }^{3}$. Contemporaine de l'émergence de la polis, l'expérience grecque de la colonisation, qui atteindra son apogée au $\mathrm{VII}^{\mathrm{e}}$ siècle, contribua non seulement à propager le modèle de la cité grecque dans plusieurs régions distantes, mais de surcroît à définir la polis comme modèle politique grec par excellence $e^{4}$. Entreprendre la rédaction d'une Politeia montre bien qu'au-delà de toutes les critiques qu'il peut adresser aux réalités politiques de son époque, Platon demeure un fervent défenseur du modèle de la polis. Il faut donc tâcher de comprendre dans quelle mesure la prise de position de Platon en faveur de la polis imprègne l'argumentaire de la Politeia.

La démarche proposée par Socrate de construire une cité en paroles aura

1. Mes plus vifs remerciements vont à Georges Leroux qui a lu et commenté une première version de cette étude. Je remercie aussi Annie Villeneuve pour sa relecture patiente du texte. Les erreurs sont évidemment les miennes.

2. Voir Leroux 2005, notamment p. 123-124 où ce dernier fournit une synthèse rapide du débat.

3. Voir la synthèse de Dougherty 1993 p. 15-27.

4. Voir Malkin 1987b p. 1. 
un terme une fois la cité fondée (IV, 427c) et c'est à ce moment que l'on pourra reprendre la recherche initiale sur la justice. On peut donc affirmer, et les pages qui suivent tenteront d'en fournir une justification, qu'au cœur de la réflexion sur la justice se trouve un questionnement préalable sur le fondement du politique, sur l'arche qui non seulement confere à la polis sa légitimité, mais lui garantit d'être juste, harmonieuse et exempte de dissension. Si la cité platonicienne s'organise autour d'une conception de la justice qui structure conséquemment la cité entière, nous aimerions observer ce qui se déroule en amont et qui légitime le philosophe dans son projet. Autrement dit, qu'est-ce que cela signifie que de fonder en paroles une cité ? Pour répondre à cette question, il faudra d'abord nous pencher sur l'expérience grecque de la fondation en portant un regard attentif sur l'exemple de la colonisation de Thourioi, qui aide à préciser le contexte historique de la République. Ensuite, il nous faudra analyser pourquoi Platon désigne Socrate comme fondateur (oikistes) et législateur de cette cité en paroles. Ces explications nous permettront alors de réfléchir aux rapports qu'instruit Platon entre la construction de sa cité et le rôle traditionnel de Delphes, en particulier l'action d'Apollon dans le processus de fondation.

\section{La fondation historique d'une cité : l'exemple de Thourioi}

Nous l'avons évoqué, le phénomène de la colonisation atteint son apogée $\mathrm{au} \mathrm{VII}^{\mathrm{e}}$ siècle, mais des colonies sont encore fondées au $\mathrm{V}^{\mathrm{e}}$ siècle, notamment Amphipolis en 437 et surtout Thourioi, vaste projet mis en avant par Périclès en $444^{5}$. Une vingtaine d'années avant la naissance de Platon, l'expérience de la colonisation demeure bien vivante à Athènes. L'exemple de Thourioi s'avère particulièrement intéressant dans notre compréhension de l'arrière-plan historique de la République, car la cité avait été pensée comme une colonie panbellénique. Or, même si ce panhellénisme n'était probablement qu'une stratégie visant à poursuivre la politique d'hégémonie impériale d'Athènes $^{6}$, il montre comment la fondation de la colonie ne s'était pas faite en raison d'une crise particulière ${ }^{7}$, mais en fonction d'un projet politique bien réfléchi ${ }^{8}$.

5. Voir Freeman 1941 et Ehrenberg 1948. À cette liste, on pourrait ajouter la fondation de Mégalopolis en 368 dont on aurait demandé à Platon de rédiger les lois, ce qu'il aurait refusé (voir Diogène Laërce, III, 23 et Élien, VH, 2, 42). Les Cyrénéens lui auraient aussi demandé de légiférer, sans plus de succès (Plu., Luc. II, 4-5 et Adprinc. inerud., 779D ; Élien, VH, 12, 30). Sur ces anecdotes, probablement fausses, voir Swift Riginos 1976 p. 191-193.

6. Ehrenberg 1948 p. 159-160.

7. La plupart des récits de colonisation s'enracinent dans différentes crises qui motivent le déplacement. Voir Dougherty 1993 p. 16-18.

8. Voir Fleming 2002 p. 6-8. Notons que la notion de panhellénisme est présentée de manière favorable par Platon dans la République (V, 469b-471b). À ce sujet, voir Morgan 1990 p. 107. 
Parmi les noms associés à la fondation de Thourioi, on note Hérodote, dont l'œuvre fait place à une réflexion nouvelle sur le politique9 et chez qui, comme l'a montré Jacqueline Bordes, on trouve la première occurrence du terme politeia ${ }^{10}$. Le nom de Thourioi est aussi associé à Hippodamos ${ }^{11}$, mais surtout à Protagoras, qui en aurait rédigé la politeia (Diogène Laërce, IX, $50)^{12}$. Cette constitution, au moins pour quelques années, fut de nature démocratique, si l'on suit le témoignage de Diodore de Sicile qui la présente

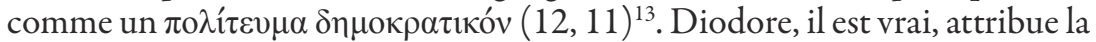
rédaction des lois de Thourioi à Charondas ${ }^{14}$, mais l'incertitude ne doit pas éclipser le fait qu'à l'époque, une réflexion sur les fondements du politique est bien en développement. On peut en retrouver des traces dans le Protagoras où le mythe que propose le sophiste s'inscrit très bien dans une réflexion sur la nature du pouvoir démocratique. On retrouve aussi de ces traces dans l'Hippias Majeur, où Platon affirme qu'Hippias jouit d'une renommée à Sparte grâce à ses discours sur les généalogies « de celles des héros comme de celles des hommes, de la fondation et de la manière dont furent instituées les premières cités » (285d, trad. Pradeau \& Fronterotta).

La fondation de Thourioi peut ainsi éclairer le projet de Platon sur plusieurs éléments. Certes, Hérodote n'apparaît pas dans la République et Protagoras n'est mentionné explicitement qu'une seule fois, dans un passage qui reprend la critique habituelle envers l'enseignement des sophistes (X, 600c). Mais la mise en scène au Pirée peut, elle, évoquer Hippodamos à qui on en devait l'aménagement. Il est par ailleurs utile de rappeler que le dialogue se déroule dans la maison de Céphale, où nous retrouvons ses trois fils Lysias, Euthydème et Polémarque, qui s'embarquèrent tous pour Thourioi à la mort de leur père. On peut donc penser raisonnablement que le souvenir de Thourioi est présent en toile de fond dans le texte de Platon. Ajoutons que le contexte colonial est d'autant plus marqué que Céphale était originaire de Syracuse, opulente colonie fondée par Corinthe. Ces deux cités trouvent d'ailleurs une place, peu enviable, dans la République, alors que

9. Voir le passage maintes fois cité de l'Enquête où Hérodote rapporte un débat entre Perses au sujet du meilleur régime (III, 80-84). L'idéal panhellénique se retrouve aussi dans son Enquête (VIII, 144) : « Il y a le monde grec, uni par la langue et par le sang, les sanctuaires et les sacrifices qui nous sont communs, nos mœurs qui sont les mêmes » (trad. Barguet).

10. Hérodote, IX, 34. Voir Bordes 1982 p. 19.

11. Hippodamos, « qui inventa la division régulière des villes et qui découpa le Pirée » aurait aussi selon Aristote rédigé une constitution (voir Aristote, Pol. II, 8, 1267b). Stobée cite d'ailleurs un Peri politeias d'Hippodamos (IV, 1, 93), même si on ne peut être certain de l'authenticité du titre. Voir Ehrenberg 1948 p. 165-166 et Bordes 1982 p. 28.

12. Voir les indications à ce sujet dans Bordes 1982 p. 30-33, ainsi que dans Untersteiner 1993 p. $19-20$.

13. Voir les commentaires de Robinson 2011 p. 119-122 et 243-245. Fleming 2002 p. 8-13, croit que Périclès est l'inspirateur principal du caractère démocratique de Thourioi.

14. Lequel est mentionné par Platon en $R$. X, 599e. 
Socrate pose les bases législatives de sa cité en paroles (III, 404d). La mise en scène peut évoquer les projets entrepris par Platon à Syracuse et qui nous sont connus par la Lettre VII. En admettant l'authenticité de la lettre, on peut en déduire que Platon a d'abord tenté de réformer le pouvoir syracusain, mais sans succès. La République semble se présenter comme une réponse à cet échec et son caractère radical s'exprime dans cette recherche de l'arche politique juste à travers la fondation en paroles d'une cité. Cette lecture sera explorée dans la section suivante.

La fondation de Thourioi fournit un élément supplémentaire pour notre compréhension de la République, car loin d'être un succès retentissant du point de vue athénien, de nombreux conflits ébranlèrent rapidement la jeune colonie. La stasis éclata en 434-433 au sujet d'une dispute sur le fondateur de la colonie, celui que l'on nomme l'« oikiste ». Cette crise ne fut résolue que par une consultation d'Apollon à Delphes. Athènes avait nommé comme « oikiste » pour l'expédition un devin du nom de Lampon qui se faisait appeler exégète. L'oracle répondit aux délégués que nul autre qu'Apollon ne devait être considéré comme fondateur. Cet oracle eut pour conséquence qu'Athènes perdit son influence sur la colonie, Thourioi entrant même en conflit avec elle en 413. L'échec de la colonisation fut attribué en large partie à Lampon l'exégète ${ }^{15}$. Socrate, qui assurément entretenait des liens avec Périclès et son entourage, a-t-il pris part à des débats concernant la jeune colonie ? Dans les Nuées d'Aristophane, il est associé aux Thouriomanteis (v. 331-332). Une scolie à ces vers nous dit : «Les Thouriomanteis ne sont pas des devins de Thourioi, mais des devins qui furent envoyés à Thourioi, ville de Sicile, pour la fonder. Dix hommes furent envoyés avec parmi eux Lampon qui était devin et qu'on appelait exégète ${ }^{16}$. $\gg$ Même si on a pu noter, avec raison, que les Nuées furent représentées en 423 , alors que Platon n'a que 4 ou 5 ans $^{17}$, il est certain que le conflit de 413 - Platon a donc environ 15 ans - a dû faire resurgir les problèmes relatifs à la fondation. Assurément cet échec marqua profondément Athènes. Même si Platon ne fréquentait probablement pas encore Socrate en 413, l'épisode lui est de toute évidence connu et, comme nous le verrons plus loin, lorsqu'il fera d'Apollon « l'exégète ancestral » (IV, 427c), il s'assurera de ne pas répéter l'erreur de Lampon.

La fondation désastreuse de Thourioi fournit un éclairage important sur l'arrière-plan historique de la République. Elle offre l'exemple d'un projet

15. Sur cet épisode, la source principale est Diodore de Sicile, XII, $10 s q$., et plus particulièrement XII, 35, 1-3. Voir aussi Malkin 1987a p. 97-101, ainsi que Graham 1964, p. 35-37.

16. Notre traduction (éd. Dübner, citée par Malkin 1987a p. 98). On se moque aussi de Lampon dans les Oiseaux, en particulier 983-988, mais si l'on se fie à Athénée, il semble avoir été pris à partie par d'autres poètes comiques comme Cratinos, Callias et Lysippe (Deipnosophistes, 8, 344e-f).

17. Piérart 1974 p. 347. 
de colonisation en fonction d'un idéal politique, et de la difficulté à réaliser cet idéal. Mais elle nous offre aussi de précieuses informations sur la manière grecque de créer une cité. Surtout, elle met en avant la fonction centrale du fondateur dans le processus de colonisation. Lampon, au cœur du conflit entre Athènes et la colonie, avait été nommé « oikiste » et Platon choisira lui aussi ce terme pour décrire Socrate.

\section{Socrate oikistes}

Rappelons brièvement de quelle manière se met en branle le développement de la cité platonicienne au livre II. Comme nous l'avons rappelé en introduction, Socrate propose de rechercher la définition de la justice dans l'ensemble plus vaste de la cité (II, 368c-369a) et cette définition sera formulée explicitement par Socrate au livre IV (433a-b). Comment, dans l'argumentaire de la République, les développements composés entre ces deux passages donnent-ils à la cité platonicienne une légitimité si importante qu'elle rende possible une compréhension de la justice, indépendamment d'un discours sur la forme du bien, qui ne sera introduit qu'au livre VI (505a)?

La réponse à cette question, croyons-nous, se trouve dans l'appel formulé par Socrate dès la mise en place de la recherche politique au livre II : «Construisons en paroles notre cité, en commençant par ses débuts $(\tau \hat{\omega}$

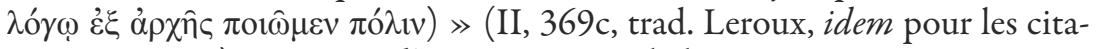
tions suivantes). Le projet d'une cité se voit balisé par trois caractéristiques précises qui en assureront la légitimité. La première concerne la fondation d'une cité dans la dimension théorique du logos. La deuxième introduit dans le champ de la réflexion politique l'importance de l'arche. Finalement, la troisième met en avant le processus de construction de cette cité. Quels liens pouvons-nous établir entre ces trois éléments?

La polis que s'apprête à construire Socrate sera produite dans la dimension du dialogue, et il faut remarquer que cette cité est destinée à rester un $\log _{0}{ }^{18}$. La possibilité ou non pour cette cité de se réaliser n'est introduite qu'au livre V (449d, mais surtout 450c). La cité fondée en paroles ne doit servir que de modèle comme le souligne Socrate en V, 472c-d :

C'était donc pour obtenir un modèle ( $\pi \alpha \rho \alpha \delta \varepsilon \dot{\gamma} \gamma \mu \alpha \tau o \varsigma)$, dis-je, que nous cherchions à savoir ce qu'est la justice en soi (...). Ce n'était donc pas dans le but précis de démontrer comment ces choses-là peuvent en venir à l'existence ( $\dot{\omega} \varsigma$

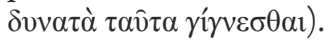

La cité théorique répond donc à un besoin préalable à tout discours sur la cité idéale : si celle-ci doit se fonder sur l'idée de justice, encore faut-il pouvoir définir cette justice. C'est pourquoi il faut retourner $\grave{\varepsilon}_{\xi} \alpha \rho \chi \eta \hat{\varphi}$, au commencement, mais surtout au principe qui doit autoriser la fondation 
de la cité ${ }^{19}$. Si la cité est correctement fondée en paroles, alors seulement pourrons-nous y voir la justice. D'ailleurs, lorsque la cité sera effectivement fondée et que l'on recherchera la justice, Socrate reviendra sur ce rapport entre arche et fondation :

Ce que nous avons établi dès le point de départ ( $\grave{\varepsilon} \xi \dot{\alpha} \rho \chi \eta \hat{\varsigma})$, alors que nous

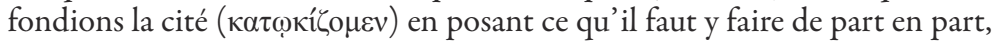
cela ou à tout le moins une certaine forme de <cette règle $>$, voilà ce qu'est à mon avis la justice. (IV, 433a)

Il faut noter toutefois l'ambiguïté qui surgit entre la fondation théorique de la cité et le caractère « poétique » de la fondation, car la poésie fut très tôt le mode d'expression privilégié des récits de fondation ${ }^{20}$. Mais l'expression

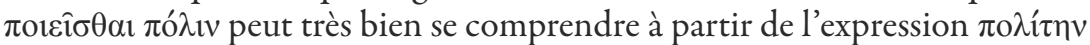
$\pi$ oıєî $\theta \alpha$, qui signifie « donner le droit de cité ». Cette expression, comme le rappelle Bordes, désignait avant l'apparition du terme de politeia son approximation la plus proche et la formule continuera d'être employée ultérieurement ${ }^{21}$. Toutefois, il ne s'agit pas seulement pour Socrate d'octroyer le droit de cité, encore moins de faire le simple récit d'une fondation imaginaire. La dimension théorique de sa construction ne l'empêche pas d'évoquer une fonction bien réelle dans la fondation, celle de l'oikiste qui non seulement fait habiter, mais aussi crée les institutions propres à la nouvelle cité.

Après le récit de la généalogie naturelle de la cité, après son gonflement et l'apparition des premières divisions, la tâche du philosophe se précise. Socrate introduit le groupe des gardiens, décrit leur formation et spécifie l'importance de « contrôler les fabricateurs d'histoires » (II, 377b). C'est alors qu'il affirme : « Adimante, dans la situation présente, nous ne sommes

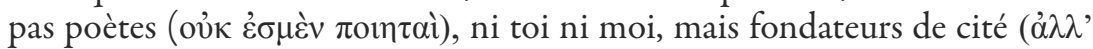

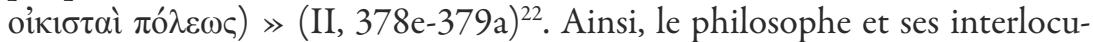
teurs se font oikistai afin de répondre aux exigences du projet commun de donner une définition de la justice (II, 386c). Par cette affirmation, Socrate entend intégrer en paroles le rôle et la figure traditionnelle du fondateur. La cité construite n'est plus d'ordre historique ou poétique : par cette nomination, Platon transforme la fonction pratique de l'oikiste en une tâche théorique et remplace l'action traditionnelle du fondateur par l'acte philosophique de la pensée et du dialogue.

Le caractère théorique de la fondation permet de mesurer la distance entre la fonction de fondateur et celle de dirigeant ${ }^{23}$. Socrate ne s'imagine

19. Narcy 2014 p. 7-8.

20. Voir Dougherty 1993 et Calame 1996.

21. Bordes 1982 p. 23, mais voir aussi p. 40-41, 51-52 et 66.

22. La dénomination de «fondateurs » qu'emploie Socrate se retrouvera aussi en VII, 519c.

23. Sur cette distinction, voir les remarques de Long 2013 p. 21-22. 
pas en philosophe-roi ni n'évoque, au cours de la fondation théorique entre les livres II et IV, la direction de la cité. Pour cause, comme nous l'avons dit, la réalisation possible de la cité n'est introduite qu'au début du livre $\mathrm{V}$ avec les deux grandes vagues que sont la présence des femmes parmi les gardiens et la communauté des femmes et des enfants $(\mathrm{V}, 457 \mathrm{bc})$. Mais c'est la troisième vague, celle du règne des philosophes $(\mathrm{V}, 473 \mathrm{ce})$ qui est présentée comme une condition sine qua non de la réalisation de la cité :

Et d'ici à ce que cela se produise, cette constitution politique que nous avons exposée dans le dialogue que nous entretenons maintenant ne pourra jamais se développer pleinement, ni voir la lumière du soleil. (V, 473e)

Peut-être ces propositions pourraient avoir l'air « ridicules et contraires à l'usage », mais seulement « si on mettait en pratique ce qu'on avance en

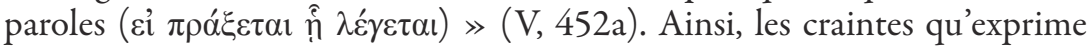
Socrate sur son discours comme « vœux pieux $\gg^{24}$ ne portent que sur ces trois vagues et non sur la cité théorique, la cité fondée en paroles qui aura permis de découvrir la justice et qui jouit pour cette raison d'une politeia «bonne et droite » $(\mathrm{V}, 449 \mathrm{a})^{25}$. Pourtant, si la thèse des philosophes-rois pose les plus grandes difficultés, le fait que Socrate s'autoproclame fondateur ne suscite aucune réaction, ni opposition de la part des interlocuteurs du dialogue. Ainsi, si nous désirons comprendre ce que signifie fonder en paroles une cité, il faut nous pencher sur la valeur du terme de « fondateur ».

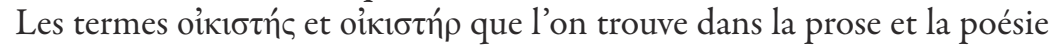
doriennes, désignent avant tout le chef de l'expédition colonisatrice ${ }^{26}$.

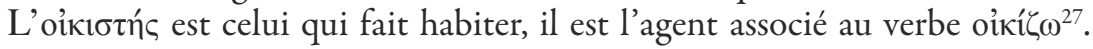
Chef de la colonie, fondateur officiel, l'« oikiste » dispose d'une autorité politique, militaire et religieuse. Il légifere en posant les bases de l'ordre social,

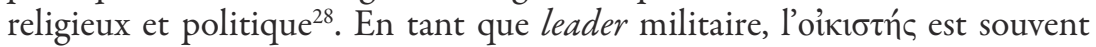

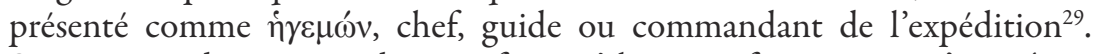

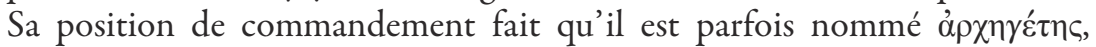
archégète, mettant l'accent cette fois sur l'idée de commencement et d'origine au cœur du procédé de fondation ${ }^{30}$. Même s'ils ne sont pas tout

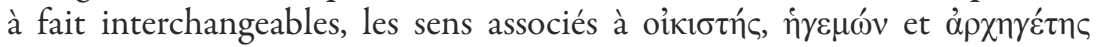
tendent à se superposer ${ }^{31}$. L'épithète d'archégète était d'abord réservée à

24. Voir V, 450d, V, 456c et VII, 540d.

25. Voir Romeri 2008 p. 29-30.

26. Casevitz 1985 p. 104.

27. Casevitz 1985 p. 104.

28. Malkin 1987a p. 89-90.

29. Malkin 1987a p. 89-90.

30. Malkin 1987a p. 243 où ce dernier propose l'étymologie suivante d'archégète : « The word has its primary connotation in the idea of beginning and origin, $\dot{\alpha} \rho \chi \eta$, and leadership

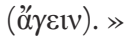

31. Malkin 1987a p. 248-249. 
Apollon $^{32}$, mais à la mort de l'« oikiste », ce dernier recevait un culte public où il devenait «l'homologue mortel du dieu $»^{33}$. Honoré en tant que héros fondateur, l'« oikiste » acquérait par le fait même lui aussi le statut d'archégète ${ }^{34}$. C'est ainsi que l'« oikiste » détenait une autorité religieuse importante. Cette autorité lui venait directement de sa nomination par Apollon et lui donnait la responsabilité d'agir et de prendre des décisions religieuses : l'institution de cultes et rites, l'indication des aires sacrées de la cité et la fondation de temples. Il était donc responsable de tout l'aspect religieux de la fondation. On saisit bien pourquoi Socrate emploie ce titre pour se décrire au moment où sera exposée la theologia, les discours sur les dieux qui devront être tenus dans la cité.

Ces quelques remarques sur la fonction de l'oíkıтń s nous conduisent directement au cœur du texte de la République: il n'y avait aucune fondation sans la nomination d'un « oikiste », ce qui invite à penser que le projet socratique d'une fondation théorique, pour pouvoir se réaliser théoriquement, a besoin d'un ou de plusieurs fondateurs. Ainsi, non seulement Socrate apparaît comme fondateur, mais aussi comme guide. Ce Socrate-fondateur devra proposer, en tant qu'« oikiste », une législation, poser les bases de la cité, poser son arche. Socrate archégète : le chef de la fondation, mais aussi le chef de la discussion, celui qui nous guide vers la cité-justice. Bref, en intégrant dans le dialogue la figure de l'« oikiste », Platon détache l'action du fondateur de l'histoire et des aléas des affaires humaines. $S$ 'il reste fidèle au modèle historique qui garantissait une fondation réussie, c'est qu'il peut intégrer en paroles ce modèle et s'assurer, par conséquent, d'une fondation idéale.

La fondation de la cité aura un terme au livre IV, lorsque Socrate affirmera à Glaucon que sa cité est « maintenant fondée » (IV, 427c). La justice s'y trouvera facilement en raison de cette fondation idéale : « Je pense que notre

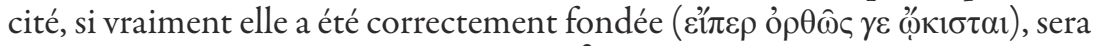

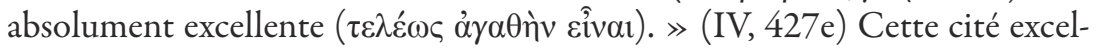
lente, parce que correctement fondée, se trouve nécessairement sage, courageuse, modérée et juste (IV, 427e). On peut donc affirmer que la démarche proposée par Socrate est couronnée de succès et si la cité est « correctement fondée », c'est que Socrate l'《 oikiste » s'est bien acquitté de sa tâche. Il faut donc mieux cerner l'activité législative du fondateur qui s'est exprimée entre le projet de fondation (II, 369c) et la fondation effective (IV, 427c).

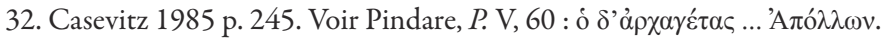

33. Detienne 1990 p. 310 . Voir aussi Casevitz 1985 p. 245-246, mais surtout Malkin 1987a p. 189-203.

34. Detienne 1990 p. 310 . Voir encore une fois le Serment des fondateurs (dans Meiggs \&

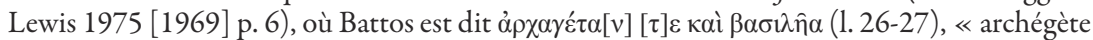

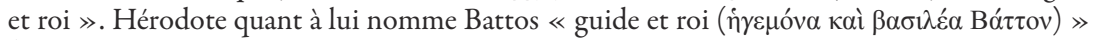
(Histoires, IV, 153), ce qui met de nouveau l'accent sur la proximité des trois termes oíkıðт́c,

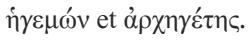




\section{Législation et tupoi}

Le rapport intrinsèque entre politeia et nomos traverse l'entièreté de la République 35 et Socrate se positionne comme législateur, au même titre que Solon, Lycurgue et Charondas (voir X, 599d-e). Il n'y a rien d'étonnant dans cette posture qui trouve son origine dans sa nomination comme « oikiste ». Toutefois, Socrate ne parle pas d'emblée de lois. Il explique à Adimante :

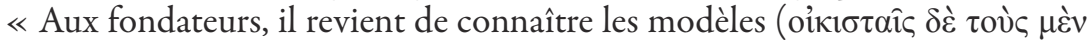

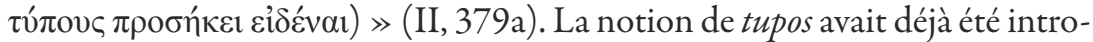
duite plus tôt dans la République pour montrer l'importance de l'éducation. Là, Socrate affirmait : « tu sais bien qu'en toute tâche, la chose la plus impor-

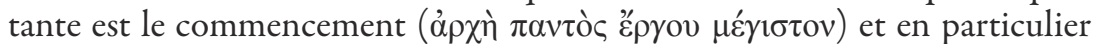
pour tout ce qui est jeune et tendre ? C'est en effet principalement durant cette période que le jeune se façonne et que l'empreinte ( $\tau \dot{\pi} \pi \mathrm{oc}$ ) dont on souhaite le marquer peut être gravée » (II, 377ab). Le rapprochement entre les deux notions d'arche et de tupos revêt un sens fort, car Socrate prétend fonder ex arches sa cité (II, 369c). Le rôle des fondateurs, s'il n'exclura pas le recours à un vocabulaire législatif, consistera à proposer des archétypes, à formuler les modèles du commencement qui garantiront dès lors à la cité en paroles cette fondation « absolument excellente » (IV, 427e). Tentons de préciser cette action.

Les premiers modèles qui sont avancés par Socrate concernent la theologia (II, 379a), dont le rôle est de préciser la norme selon laquelle seront produits les récits poétiques ${ }^{36}$. Victor Goldschmidt a déjà montré comment cette theologia représentait une division traditionnelle de la muthologia qui avait pour objet la représentation des dieux ${ }^{37}$. Les modèles que propose Socrate tentent de purifier ces représentations traditionnelles. L'action de l'« oikiste » se présente dès lors comme un geste rationnel, puisque, faut-il le rappeler, c'est au logos que fait appel Socrate pour fonder sa cité. L'explication de ce premier modèle trouve sa conclusion en II, 380c :

Cette loi sera donc, repris-je, la première des lois ( $v o ́ \mu \omega v)$ relatives aux dieux

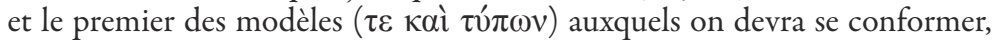
tant les conteurs dans leurs récits que les poètes dans leurs poèmes : que le dieu n'est pas la cause de toute chose, mais seulement des biens.

Les modèles que doivent connaître les fondateurs ont donc le statut de lois primordiales, ils sont nécessaires à la cité « si celle-ci doit être régie par

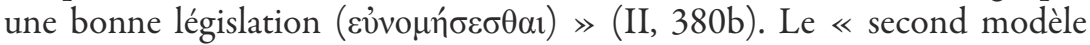

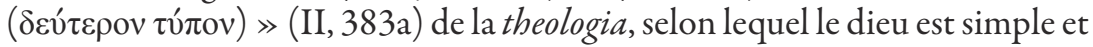
véridique, rencontrera l'accord d'Adimante : «Pour ma part, dit-il, je m'ac-

35. Lane 2013 p. 104.

36. Sekimura 1999 p. 66.

37. Goldschmidt 1950 p. 27 et 30. 


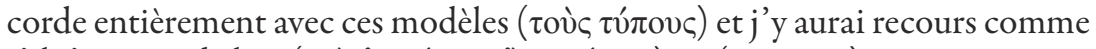

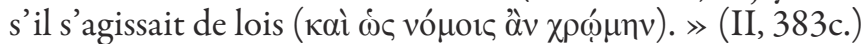

Ce vocabulaire législatif, où se croisent nomos et tupos, se retrouve abondement au livre III $^{38}$. À propos des règles concernant la musique et la poésie, on mentionne le « modèle (

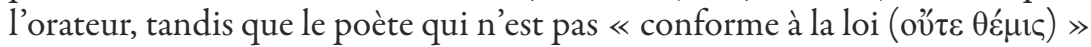
(III, 398a) sera expulsé de la cité. Ensuite, en III, 398b, on exige un poète

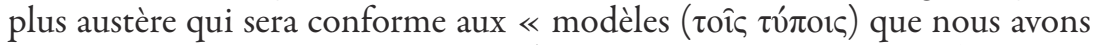

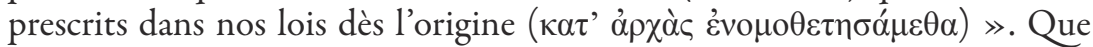
la parole du poète soit chantée ou non, il n'y a aucune différence « en ce

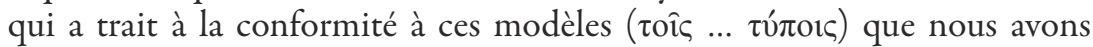
proposés tout à l'heure » (III, 398d). Cette parole devra favoriser les vertus de courage et de modération. Il faudra donc fuir les excès liés à l'amour et Socrate explique que « dans la cité que nous sommes en train de fonder ('ُ $\mathrm{v}$

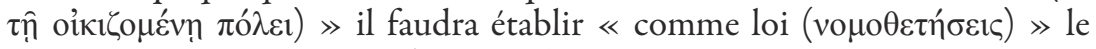
refus de tout contact sexuel (III, 403b).

En ce qui concerne le corps, il faudra laisser à « l'art de la gymnastique la tâche de préciser les soins du corps, en nous contentant d'indiquer pour

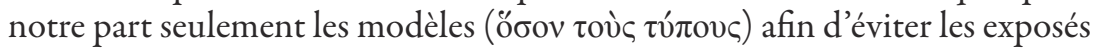
trop élaborés »(III, 403de). Ces modèles prendront là aussi quelquefois la forme de lois. Socrate parle, en effet, d'établir « par voie de législation

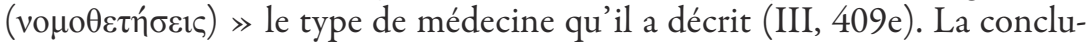
sion de cette section reviendra sur la notion de modèle : « voilà donc quels

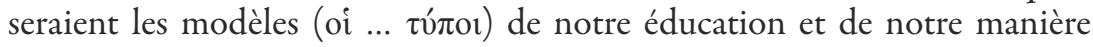
d'élever les enfants » (III, 412b). Suivront quelques remarques sur l'habitat des gardiens et leur mode de vie, qui seront eux aussi soumis à des « disposi-

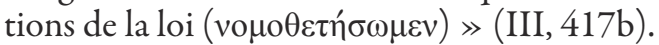

Il faut pour comprendre cette idée retourner au premier emploi de tupos dans la République. Il permet de comprendre comment un tupos suppose une empreinte qui perdurera dans le temps. Ainsi, le rôle de l'« oikiste »-législateur est ici de fournir l'impulsion initiale à la politeia. Ce rapport est présenté au livre IV où Socrate explique :

Et sans doute, dis-je, une constitution politique ( $\pi$ o $\lambda ı \tau \varepsilon i ́ \alpha)$, à condition d'avoir au départ une bonne impulsion ( son mouvement de croissance de façon concentrique. La manière d'élever et d'éduquer les enfants, si elle demeure honnête, produit des natures bonnes. (IV, 424a)

38. Une liste exhaustive du vocabulaire législatif dans la République se trouve dans Lane 2013 p. 105-108. 
L'important est d'éduquer dans le « respect de la loi (Eủvouíav) » (IV, 425a) et non de légiférer sur tout ce qui mériterait des $\operatorname{lois}^{39}$. Ainsi, Socrate n'a pas à légiférer sur tout, mais doit néanmoins fournir les modèles de départ pour la politeia qui est maintenant nommée comme telle (voir aussi III, 397e et III, 412a). C'est le caractère radical des modèles, leur archétype législatif qui peut garantir une fondation juste et suffisante.

L'usage de la notion de tupos pour désigner les législations fondamentales se comprend assurément en fonction du caractère primordial de l'éducation dans la fondation de la cité juste. Même si tupos relève d'abord d'un contexte plastique où il désigne « l'empreinte en creux (imprimée) ou en saillie (repoussée) que laisse la "frappe" d'une matrice, l'emblème figuré sur cette matrice et que la "frappe" reproduira $\gg^{40}$, Socrate l'emploie dans un contexte éducatif qui est d'emblée politique. Tupos aide ainsi à penser le rapport psycho-politique au cœur de la démarche de fondation des livres II-IV, car il relie l'empreinte inscrite dans l'âme des gardiens au caractère législatif de leur paideia. Ces «tupoi législatifs ${ }^{41}$ sont les modèles à partir desquelles toutes les lois ultérieures devront êtres établies. Socrate l'exprime très clairement en V, 458c lorsqu'il souligne que les gardiens devront obéir aux lois primordiales de la fondation, mais que lorsqu' ils devront eux aussi légiférer, ils devront le faire en « imitant ( $\mu \mu о \nu \mu \varepsilon ́ v o v \varsigma)$ ces lois ». Les lois proposées par Socrate ne sont donc ici que le mode d'expression légal de tupoi éducatifs. En IV, 445e, Socrate précise d'ailleurs que le nombre de dirigeants importe peu, tant et aussi longtemps que ne sont pas bouleversées les « lois

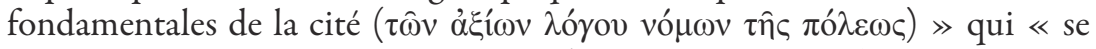

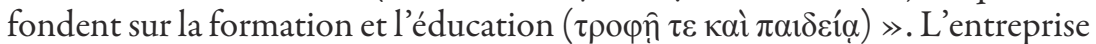
législative des fondateurs est ainsi et d'abord une entreprise éducative.

En proposant des modèles législatifs, Socrate fonde la cité à l'intérieur de laquelle il sera possible de découvrir une définition de la justice. Cette définition, qui n'interviendra à proprement parler qu'en IV, 433a, est en fait déjà présente, en raison précisément de ces modèles législatifs. Nous la trouvons au livre III, 406c : « Partout où existent des lois excellentes, une tâche propre

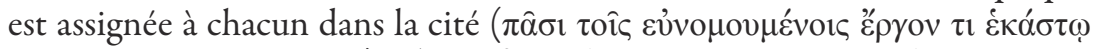

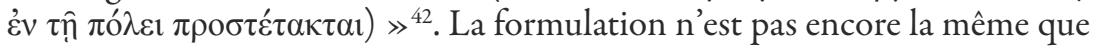

39. Socrate renonce à légiférer sur des points trop détaillés concernant les règles de civisme ou encore sur les questions économiques (IV, 425b-e).

40. Roux 1961, p. 5.

41. L'expression est de Sekimura 1999, p. 69.

42. En IV, 433a, que nous avons cité supra, Socrate fait allusion à ce passage : « Ce que

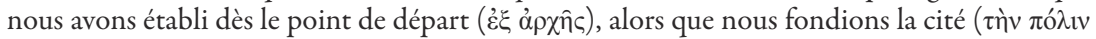

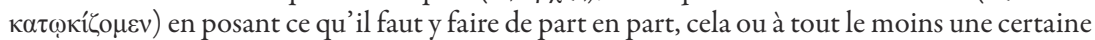
forme de <cette règle>, voilà ce qu'est à mon avis la justice. Nous avons posé en effet, et nous en avons parlé souvent si tu t'en souviens, que chacun devait exercer une fonction particulière parmi celles qui concernent la cité. » 
celle qui sera donnée plus loin, mais néanmoins, ce passage nous indique clairement comment l'eunomia d'une cité implique la division des tâches ${ }^{43}$. Dans le cadre qui nous intéresse, il est facilement compréhensible que Socrate affirme cette importance de la bonne législation puisque son rôle d'oikiste le

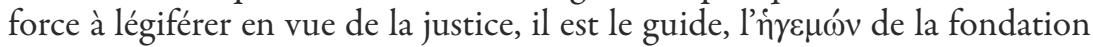
idéale. Il reviendra plus loin sur cette même idée de la tâche propre, mais cette fois-ci en lien direct avec la fondation. Il faudra convaincre les gardiens et les auxiliaires, en vue du bonheur de la cité, pour « qu'ils deviennent les

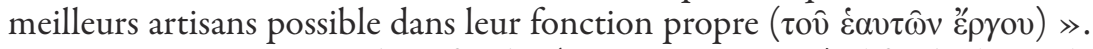

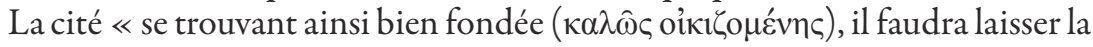
nature accorder à chacun des groupes la possibilité d'avoir part au bonheur » (IV, 421c, trad. Leroux légèrement modifiée). Une législation qui oriente chaque groupe vers sa tâche propre aura ainsi la chance de se réaliser dans une belle fondation, elle-même garante de la séparation des tâches (ce qui deviendra plus loin, après la fondation de la cité, le principe de justice).

La fonction de l'« oikiste » semble pour le moment la seule à permettre à Socrate de guider la discussion vers un paradigme de la justice. La raison du philosophe-fondateur en paroles apparaît avoir assez d'autorité pour définir des modèles qui viendront encadrer une cité juste. En effet, aucune cité actuelle ne convient véritablement à la philosophie, sinon « la constitution

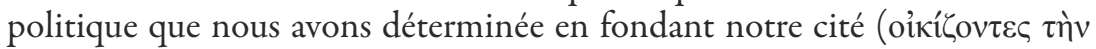
$\pi$ ó $\lambda v v) \gg(\mathrm{VI}, 497 \mathrm{c})$. Selon Socrate,

il devrait toujours y avoir au cœur de la cité quelque fonction dépositaire

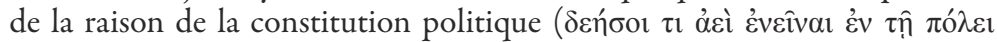

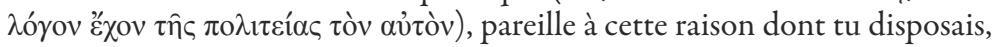

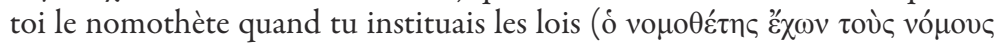

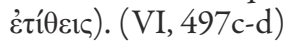

Toutefois, il ne faut jamais oublier que l'oikiste ne peut s'autoriser lui-même. L'épisode du conflit à Thourioi entourant Lampon nous rappelle que l'autorité de l'oikiste provient directement d'Apollon et c'est pourquoi, avant de compléter la fondation de sa cité en paroles, Socrate évoquera à son tour l'autorité du dieu de Delphes.

\section{Delphes et la fondation}

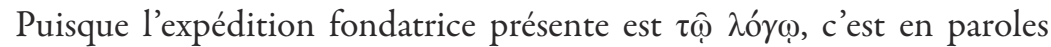
qu'il faut consulter Apollon. En IV, 427b, Socrate se rend auprès du Pythien, allant chercher du coup l'autorité nécessaire à l'achèvement de son œuvre d'《 oikiste » :

Mais que peut-il bien nous rester, dit-il, qui puisse encore faire l'objet de

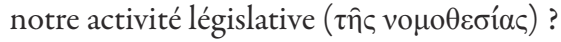


- Pour nous, répondis-je alors, il ne reste rien, mais pour l'Apollon de Delphes, il reste les législations les plus essentielles, les plus belles et les

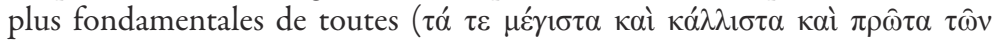
vo $\mu \mathrm{o} \theta \varepsilon \tau \eta \mu \alpha ́ \tau \omega v)$. (IV, 427b)

Remarquons d'abord, en continuité avec ce qui a été dit précédemment, que l'activité législative des « oikistes » est maintenant terminée. En revanche, plusieurs lois concernant la vie religieuse de la cité sont laissées à l'Apollon de Delphes. Il ne s'agit pas de lois anodines, plutôt des lois les plus fondamentales, $\pi \rho \omega \hat{\tau} \alpha$, c'est-à-dire celles qui sont au fondement de la cité : les premières des lois, premières dans l'ordre chronologique, mais aussi premières en importance. À la fois les plus élevées, mais aussi les plus ancrées au sol, celles qui forment les fondations de la future législation, les lois préalables à toutes les autres : les législations du commencement de la cité. Déjà, nous avons souligné « qu'en toutes choses la plus importante est

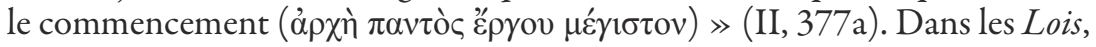
Platon renchérit :

Un de nos proverbes dit en effet que le commencement ( $\dot{\alpha} \rho \chi \eta ́)$ est la moitié

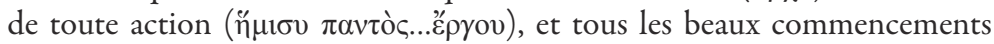

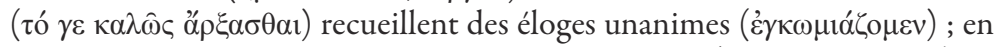

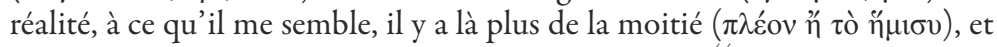
un beau commencement n'a jamais reçu assez d'éloges ${ }^{44}$.

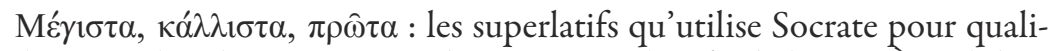
fier l'activité législative nous conduisent tous à l'arche de la cité. À Delphes, Socrate vient chercher le commencement et le principe de sa cité, son arche, et pour ce faire consulte l'Archégète. L'accord final, la sanction d'Apollon vient ainsi donner l'autorité nécessaire aux « oikistes » pour compléter la fondation de la cité théorique, fondation qui suivra immédiatement ce passage : « Mais alors, fils d'Ariston, dis-je, ta cité serait donc maintenant fondée $(\grave{\omega} \iota \kappa \imath \sigma \mu \varepsilon ́ v \eta) \gg(I V, 427 \mathrm{c})$.

La présence de Delphes dans ce passage ne devrait pas nous surprendre, nous avons déjà vu que l'oikistes se devait d'aller consulter l'oracle de Delphes avant de lancer l'expédition vers la future colonie ${ }^{45}$. Les « oikistes » étaient le plus souvent délégués par les métropoles engagées dans une expédition colonisatrice. Dans ce contexte, les fondateurs vont chercher à Delphes « une autorisation divine » ${ }^{46}$ à leurs projets et c'est aussi cette autorité que va consulter Socrate. À Apollon reviennent

les législations relatives aux fondations des temples sacrés, aux sacrifices et à toutes les choses qui concernent le culte des dieux, des démons et des héros. Aussi ce qui concerne les sépultures des défunts et tout ce qui est requis

44. Lois, VI, 753e-754a. Trad. Des Places. Le proverbe se retrouve aussi dans $R$. II, 377a. 45. Parke \& Wormell 1956 p. 49. 46. Parke \& Wormell 1956 p. 50. 
pour servir ceux qui sont là-bas et leur assurer la sérénité. Les choses de ce genre, nous ne les connaissons pas nous-mêmes quand nous fondons une cité

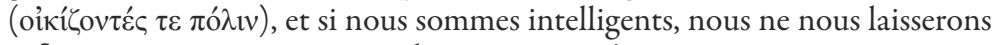
influencer par personne, pas plus que nous n'aurons recours à un exégète

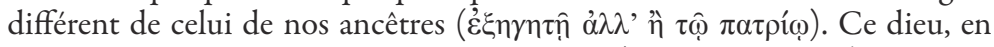

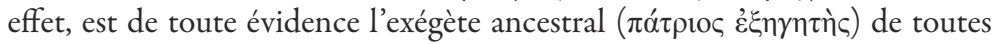
ces questions pour tous les hommes ( $\pi \hat{\alpha} \sigma \mathrm{v} v \dot{\alpha} v \theta \rho \omega ́ \pi \mathrm{s} \varsigma)$, lui qui, siégeant au

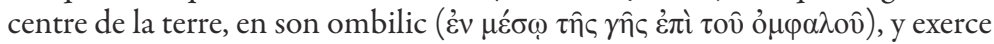

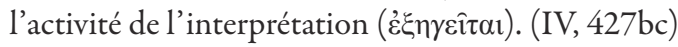

L'autorité en matière religieuse, lorsqu'il est question de fonder une cité, se trouve à Delphes et Socrate, révélant son ignorance à ce sujet, ne fera pas exception à cette règle. Ainsi, même les tupoi de la theologia élaborés plus tôt et qui concernaient la façon dont les dieux devaient être représentés, sont soumis à l'autorité d'Apollon, car seul ce dieu peut nous indiquer le chemin à suivre sur tout ce qui concerne le culte des dieux. Ses lois, en raison de leurs valeurs fondamentales, rendent possibles toutes les autres, puisqu'elles se situent au commencement ${ }^{47}$.

La cité en paroles que fondent Socrate et ses interlocuteurs est donc placée entre les mains du Pythien. Le rôle d'Apollon, tel que mentionné en IV, 427b-c, concerne dans un premier temps les lois à propos de l'établisse-

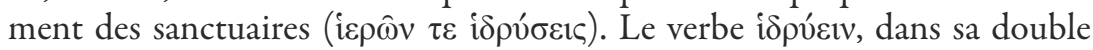
signification de fonder et de consacrer, délimite l'espace religieux et l'espace profane. Historiquement, il s'agissait d'un des premiers actes de la fondation $^{48}$. En expliquant le rôle traditionnel de l'« oikiste », Marcel Detienne a fait ressortir la signification précise de cet acte de délimitation :

Le Fondateur autorisé par Apollon procède à la découpe du territoire. Il consacre et délimite les espaces réservés aux dieux ; autels, sanctuaires, temples. Il trace, il balise les espaces réservés à la vie publique, aux assemblées, aux lieux de décision, aux habitations distribuées le long des voies d'accès. Autant d'opérations qui relèvent de l'art de découper, de temnein en même temps que celui d'établir, d'asseoir, de consacrer, d'bidruein ${ }^{49}$.

De toute évidence, nulle part dans la République, Socrate ne procède à un tel découpage ${ }^{50}$. Au contraire, il demande l'expertise législatrice d'Apollon sur ces questions. Toutefois, ce passage précède la fondation et ainsi, la

47. Delphes demeurera une autorité fondamentale lorsqu'il sera question de législation. Voir notamment V, 461e et V, 469a. Voir aussi VII, 540c.

48. Malkin 1987 b p. 339.

49. Detienne 1990 p. 310.

50. Le partage du territoire deviendra dans les Lois un aspect central dans la construction théorique de la cité. Voir par exemple V, 745b-e. Il est à noter que les Lois intègrent aussi, mutadis mutandis, le modèle grec de la fondation. Clinias est explicitement décrit comme un « oikiste » et Delphes y est plus présent que dans tous les autres dialogues de Platon. Toutefois, l'étude du rôle de Delphes dans les Lois commanderait à elle seule une étude séparée. 
consultation en paroles de Socrate concerne peut-être la manière dont devra être découpée la cité, et particulièrement ses aires sacrées. Découpage certes théorique, mais qui permet de compléter la fondation de la cité. Il semble à cet effet important de voir comment s'articule la proximité entre l'Apollon de Delphes avec l'action de fonder (oíí̧ rentes aires de la cité. Platon intègre à nouveau en paroles ce qui pour les Grecs garantissait une fondation parfaite. Par cette consultation en paroles, il transporte une fois de plus dans le domaine du logos l'action historique de la fondation et cette activité lui permet de fonder en paroles une cité idéale.

\section{Apollon exégète}

Précisons le rôle traditionnel d'Apollon dans le processus de fondation. «C'est sur les pas de Phoibos qu'on trace l'enceinte des cités; Phoibos se plaît à leur établissement, et sa main en bâtit les fondements ${ }^{51}$. $\gg C^{\prime}$ 'est ainsi que Callimaque parle d'Apollon, et il ne s'agit pas d'une originalité propre au poète. Théognis de Mégare écrivait déjà : « Puissant Phoibos, c'est toi qui as élevé les murs de notre citadelle ${ }^{52}$. » Apollon est celui qui guide, qui découpe, « le couteau à la main », le territoire de la cité : «C'est un conquérant, un marcheur : partout où il fait un pas, il prend pied, il taille, il coupe, il délimite, il fait du territoire ${ }^{53}$. »

L’Apollon fondateur de cités est associé à deux épithètes fondamentales, archégète et exégète, toutes deux étant par ailleurs intrinsèquement liées :

La catégorie d'archégète associe à l'image de celui qui conduit la valeur double d'archein : commencer et commander, l'origine dans l'ordre temporel avec la primauté dans l'ordre social. En principe, chacun des dieux peut prétendre au titre d'archégète comme à celui de puissance poliade, première dans le panthéon d'une cité singulière. C'est pourtant un seul parmi les Olympiens qui semble avoir le monopole du statut d'archégète : Apollon ${ }^{54}$.

Le véritable fondateur, le fondateur idéal, est Apollon et l' « oikiste » agit en imitant son action. Apollon archégète représente donc d'emblée le dieu de l'arche politique. Quelle autre divinité serait mieux placée dans la situation présente pour présider à la fondation de la cité idéale en paroles ? Apollon garantit la fondation harmonieuse, il permet d'éviter la stasis ${ }^{55}$. L'exemple de Thourioi, encore une fois, nous rappelle que la consultation entourant

51. Callimaque, Ap. 55-57. Trad. E. Cahen.

52. Poèmes élégiaques, 773. Trad. J. Carrière. Rappelons que c'est à Apollon et à Poséidon que l'on doit les murailles de Troie. Voir Iliade, VII, 452-453 et Pindare, Olympiques, VIII, 30-52.

53. Detienne 1998 p. 235. Nous faisons évidemment référence au titre de son livre Apollon le couteau à la main.

54. Detienne 1998 p. 92.

55. Voir Théognis, Poèmes élégiaques, 780-782 ainsi que Detienne 1998 p. 93. 
l'« oikiste » de la cité avait été mise de l'avant pour répondre à la stasis qui divisait la cité. Harmonie de la cité, unité de la cité : ces thèmes se retrouvent tous dans la République et constituent un point central de la philosophie politique de Platon $^{56}$. Cette unité est rendue possible par l'universalité d'Apollon qui s'adresse à toute l'humanité : «Ce dieu, en effet, est de toute

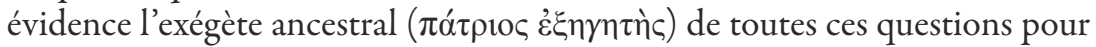
tous les hommes ( $\pi \hat{\alpha} \sigma \mathrm{v} v \dot{\alpha} v \theta \rho \omega ́ \pi \mathrm{orc})$. » (IV, 427c) Apollon apparaît donc, en tant qu'archégète, comme un personnage essentiel à la fondation idéale, il en assure la cohésion et l'harmonie.

Cette vertu d'archégète est directement liée à celle d'exégète. L'Apollon exégète, l'exégète ancestral ( $\pi \alpha$ ó celui qui dirigera la fondation. Encore une fois, citons Marcel Detienne :

Dans le composé d'où vient le mot « exégète », le sens de gouverner se maintient à côté de celui d'exposer, de développer un enchaînement de mots, de tracer un chemin de paroles comme il convient à un dieu siégeant en un oracle ainsi fondé s. $^{57}$.

L'Apollon exégète de Platon est celui qui non seulement, en tant qu'archégète, trace le chemin de la cité, mais qui, en tant qu'exégète, trace en paroles ce chemin. C'est un dieu de l'épyov politique lorsqu'il trace l'enceinte des cités, lorsqu'il délimite le territoire, mais c'est aussi un dieu du $\lambda$ ó ${ }^{\circ} \varsigma_{\text {c }}$ politique lorsqu'il délimite en paroles cet espace. Or la fondation présente est une fondation théorique. On comprend alors pourquoi Platon met l'accent

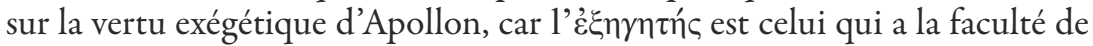

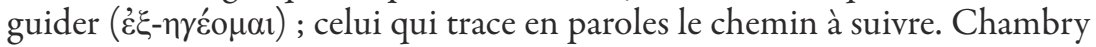
traduit d'ailleurs ce passage en insistant sur la vertu de guide d'Apollon : «... ce dieu, interprète traditionnel de la religion, s'est établi au centre et au nombril de la terre pour guider le genre humain. $\gg$ Nul autre qu'Apollon ne doit guider l'entreprise fondatrice. Nul autre ne pourrait tracer en paroles une fondation théorique. Nul autre qu'Apollon ne peut être nommé exégète, comme l'oracle l'a rappelé à Lampon. Cet Apollon devient le guide théorique de Socrate, celui qui aide à la fondation, qui la guide vers son arche de justice, participant ainsi à l'idéalité de la fondation.

Socrate spécifie qu'Apollon, à Delphes, siège sur l'omphalos au centre de la terre :

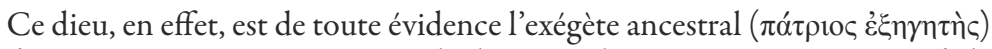
de toutes ces questions pour tous les hommes, lui qui, siégeant au centre de la

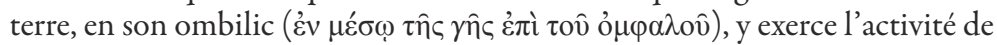

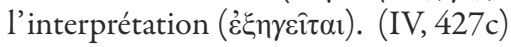

La richesse symbolique du sanctuaire de Delphes est ici présentée à travers

56. Voir par exemple IV, 422e-423a ou encore V, 470d-e.

57. Detienne 1998 p. 87. 
le double aspect de nombril et de centre qu'on ne saurait épuiser en quelques lignes. Signalons que Platon semble mettre l'accent sur le caractère central de l'omphalos, puisque lui est associée la valeur de centre ( $\dot{\varepsilon} v \mu \varepsilon \dot{\varepsilon} \sigma \omega)$. En ce sens, Platon insiste sur un élément commun de la mythologie delphique. Pindare associait déjà l'omphalos au centre : « l'omphalos qui marque le centre ( $\mu \varepsilon ́ \sigma o v$ ó $\mu \varphi \alpha \lambda$ òv) de la terre boisée $\gg^{58}$, association évidente aussi chez Eschyle qui parle de Pythô en tant que « nombril-centre $(\mu \varepsilon \sigma o \mu \varphi \alpha ́ \lambda o r \varsigma) ~ »{ }^{59}$. Nous retrouvons le même terme composé chez Euripide dans l'Oreste, à propos de Delphes « où se trouve, dit-on, l'emplacement secret de l'ombilic du monde $(\mu \varepsilon \sigma o \mu \varphi \alpha ́ \lambda o r \varsigma) ~{ }^{60}$. Ce terme composé devient dans la tragédie épithète du sanctuaire de Delphes ${ }^{61}$, ce qui prouve à quel point la valeur centrale de l'omphalos est une notion commune.

Toutefois, comme le note Alain Ballabriga, la notion d'omphalos ne recouvre pas parfaitement celle de meson.

En tant que point de fusion des niveaux cosmiques, l'ombilic fonctionne sur un plan principalement vertical, même si secondairement il peut servir à orienter un espace circulaire et horizontal, tandis que la notion de meson renvoie à celle de « milieu » d'un espace humain ou cosmique potentiellement géométrisable ${ }^{62}$.

L'omphalos devient par le fait même centre de communication entre le dieu et le philosophe qui vient y chercher une exégèse. Delphes apparaît donc comme le lieu où la relation verticale entre guide et guidé peut s'effectuer, où l'aspect théorique de l'arche tant recherchée peut enfin éclore ; où le philosophe-fondateur peut aller chercher une autorité transcendante qui garantira une fondation idéale. La notion de meson évoque quant à elle la valeur panhellénique du sanctuaire : Apollon, exégète ancestral, est le guide de la nation grecque dans son entièreté, il est même le guide pour tous les humains ${ }^{63}$.

\section{Conclusion}

Nous avons cherché dans cette étude à comprendre ce que pouvait signifier fonder en paroles une cité. Cette proposition que formule Socrate s'accompagne d'un certain nombre d'actions intimement associées au rôle de fondateur qu'il s'attribue au livre II. Dans l'argumentaire de la République, la proposition philosophique rejoint et intègre le modèle historique de la fondation. Pour le dire en un mot, le choix de la polis implique donc le

58. Pindare, $P$ IV, 74 .

59. Eschyle, Th. v. 747.

60. Euripide, Or. v. 331.

61. Chantraine 1968-1980 s.v. ỏ $\mu \varphi \alpha \lambda o ́ c$.

62. Ballabriga 1986 p. 12.

63. Voir Adam 1902 vol. 1, p. 222-223 et Nettleship 1951[1898] p. 142. 
choix de Delphes qui assurait traditionnellement la réussite de tout projet de fondation. L'action des livres II-IV reprend ainsi le schéma traditionnel : nomination d'un « oikiste », législations primordiales, découpage des aires sacrées et culte d'Apollon exégète. On retrouve là, mutadis mutandis, le schéma politique de la mission socratique telle que formulée dans l'Apologie de Socrate. Dans un cas comme dans l'autre, l'activité philosophique se voit légitimée par le recours à Delphes. Que Platon se réclame d'Apollon pour fonder une cité apparaît donc tout à fait cohérent avec la manière grecque de fonder des cités.

Au-delà du modèle historique, c'est la dimension théorique qui est convoquée chez Platon. Était-il vraiment nécessaire pour lui de conserver ce modèle historique ? Clairement, et même si la proposition philosophique de la République se révèle être une critique radicale de la polis athénienne, il n'en demeure pas moins que Platon n'a pas voulu imaginer sa cité indépendamment des contraintes religieuses qui entouraient tout projet de fondation. On ne peut donc pas s'étonner que la réflexion politique naissante ne se soit pas entièrement détachée des conditions qui l'ont vue naître. Le modèle de la polis, à tous les égards, est directement relié au sanctuaire de Delphes ${ }^{64}$. Par contre, en transposant l'ergon politique dans le champ du logos, Platon transformait radicalement cette pratique, car à partir du moment où la réflexion se détache de la pratique, c'est tout un nouveau champ d'investigation philosophique qui naît. Platon utilise la fondation en tant que modèle et cadre conceptuel dans l'élaboration d'une réflexion philosophique sur la cité excellente, mais cette utilisation permet à son tour une réflexion originale sur l'acte politique primordial, celui de la création d'un espace politique.

64. Fontenrose 1978 p. 5 souligne d'ailleurs que le déclin de Delphes se déroule parallèlement au déclin de la cité. 


\section{BIBLIOGRAPHIE}

Adam, J. 1902 (éd.) : The Republic of Plato, edited, with critical notes, commentary and appendices, Cambridge, 1902.

Ballabriga, A. 1986 : Le Soleil et le Tartare : l'image mythique du monde en Grèce archaïque, Paris, 1986 (Recherches d'histoire et de sciences sociales, 20).

Barguet, A. 1985 (trad.) : Hérodote, L'Enquête, texte présenté, trad. et annoté, Paris, 1985 (Collection Folio).

Bordes, J. 1982 : Politeia dans la pensée grecque jusqu'à Aristote, Paris, 1982 (Collection d'études anciennes).

Cahen, É. 1922 (trad.) : Callimaque, Hymnes, Épigrammes, Les Origines, Hécalé, Iambes, Poèmes lyriques, Paris, 1922 (Collection des Universités de France).

CALAME, C. 1996 : Mythe et histoire dans l'Antiquité grecque : la création symbolique d'une colonie, Lausanne, 1996 (Sciences humaines).

Carrière, J.-Cl. 1948 (éd.) : Théognis de Mégare, Poèmes élégiaques, Paris, 1948 (Collection des Universités de France).

Casevitz, M. 1985 : Le vocabulaire de la colonisation en grec ancien : étude lexico-

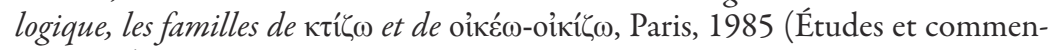
taires, 97).

Chantraine, P. 1968-1980 : Dictionnaire étymologique de la langue grecque : histoire des mots, achevé par J. Taillardat, O. Masson \& J.-L. Perpillou, Paris, 1968-1980.

Des Places, É. 1951 (éd.) : Platon, Les Lois, livres III-VI, texte établi et traduit, Paris, 1951 (Collection des Universités de France).

Detienne, M. (dir.) 1990 : Tracés de fondation, Leuven, 1990 (Bibliothèque de l'École des hautes études. Section des sciences religieuses, 93).

- 1998 : Apollon, le couteau à la main : une approche expérimentale du polythéisme grec, Paris, 1998 (Bibliothèque des sciences humaines).

Dougherty, C. 1993 : The Poetics of Colonization : from City to Text in Archaic Greece, New-York/Oxford, 1993.

Dübner, F. 1969 (éd.) : Scholia Graeca in Aristophanem : cum Prolegomenis Grammaticorum, Varietate Lectionis Optimorum Codicum Integra, Ceterorum Selecta, annotatione criticorum item selecta, cui sua quaedam inseruit, Hildesheim, 1969 [1877].

Ehrenberg, V. 1948 : «The Foundation of Thurii », The American Journal of Philology, 69/2 (1948), p. 149-170.

Fleming, D. 2002 : « The Streets of Thurii : Discourse, Democracy and Design in the Classical Polis », Rhetoric Society Quarterly, 32/3 (2002), p. 5-32.

Fontenrose, J. 1978 : The Delphic Oracle : its Responses and Operations With a Catalogue of Responses, Berkeley/Los Angeles/London, 1978.

Freeman, K. 1941 : « Thourioi », Greece \& Rome, 10/29 (1941), p. 49-64.

Goldschmidt, V. 1950 : « Théologia », Revue des études grecques, 63/294 (1950), p. 20-42.

Graham, A. J. 1964 : Colony and Mother City in Ancient Greece, Manchester, 1964.

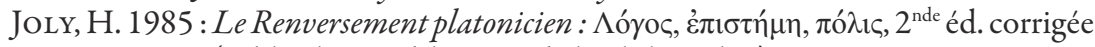
Paris, 1985 (Bibliothèque d'histoire de la philosophie). 
LANE, M. 2013 : « Founding as legislating : the figure of the lawgiver in Plato's Republic », dans N. Notomi \& L. Brisson (éd.), Dialogues on Plato's Politeia (Republic) : selected papers from the Ninth Symposium Platonicum, Sankt Augustin, 2013, p. 104-114 (International Plato studies, 31).

Leroux, G. 2002 (trad.) : Platon, La République, trad. inédite, introd. et notes, Paris, 2002 (G. F., 653).

- 2005 : «La tripartition de l'âme : politique et éthique de l'âme dans le livre IV », dans M. Dixsaut (éd.), Études sur la République de Platon, I, De la justice: éducation, psychologie et politique, Paris, 2005, p. 123-147 (Tradition de la pensée classique ).

Long, A. 2013 : «The political art in Plato's Republic », dans V. Harte \& M. Lane (éd.), Politeia in Greek and Roman philosophy, Cambridge/New York, 2013, p. 15-31.

Malkin, I. 1987a : Religion and Colonization in Ancient Greece, Leiden, 1987 (Studies in Greek and Roman religion, 3).

- 1987b : « La place des dieux dans la cité des hommes : le découpage des aires sacrées dans les colonies grecques », Revue de l'histoire des religions, 204/4 (1987), p. 331-352.

Meiggs, R. \& D. Lewis 1975 (éd.) : A Selection of Greek Historical Inscriptions to the End of the Fifth Century B.C., reprinted with corrections, Oxford, 1975 [1969].

Morgan, M. L. 1990 : Platonic Piety : Philosophy and Ritual in Fourth-Century Athens, New Haven/London, 1990.

Narcy, M. 2014 : «Cité naturelle et cité juste dans la République de Platon », Bulletin de la Société française de Philosophie, 108/4 (2014), p. 5-40.

Nettleship, R. L. 1951 : Lectures on the Republic of Plato, London, 1951 [édité pour la première fois sous le titre : Philosophical Lectures and Remains, 2, 1898].

Parke, H. W. \& D. E. W. Wormell 1956 : The Delphic Oracle, vol. I, The History; vol. II, The Oracular Responses, Oxford, 1956.

PIÉRART, M. 1974 : Platon et la cité grecque : théorie et réalité dans la constitution des Lois, Bruxelles, 1974 (Mémoires de la Classe des lettres. Collection in-8 ; Académie royale de Belgique, $2^{\mathrm{e}}$ série, 62, 3).

Pradeau, J.-F. \& F. Fronterotta 2005 (trad.) : Platon, Hippias majeur, suivi de Hippias mineur, trad. inédites, introd. et notes, Paris, 2005 (G. F., 870).

Robinson, E. W. 2011 : Democracy Beyond Athens : Popular Government in the Greek Classical Age, Cambridge, 2011.

Romeri, L. 2008 : « La cité idéale de Platon : de l'imaginaire à l'irréalisable », Kentron, 24 (2008), p. 23-34.

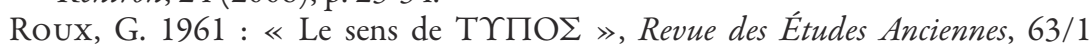
(1961), p. 5-14.

Sekimura, M. 1999 : « Le statut du tupos dans la République de Platon », Revue de philosophie ancienne, 17/2 (1999), p. 63-90.

Swift Riginos, A. 1976 : Platonica : the Anecdotes Concerning the Life and Writings of Plato, Leiden, 1976 (Columbia Studies in the Classical Tradition, 3). 
Untersteiner, M. 1993 (trad.) : Les sophistes, t. 1, trad. de l'italien et présenté par Alonso Tordesillas, préf. de Gilbert Romeyer Dherbey ; $2^{\text {nde }}$ éd. revue et notablement augmentée avec un appendice sur « Les origines sociales de la sophistique », Paris, 1993 (Bibliothèque d'histoire de la philosophie). 